\title{
Synthesis and study of complexes of the novel Russian antiviral drug Camphecene with pentacyclic triterpenes of licorice
}

\author{
S.S. Khizrieva ${ }^{*}$ E. V. Vetrova, S. N. Borisenko, \\ E. V. Maksimenko, N. I. Borisenko \\ Research Institute of Physical and Organic Chemistry, \\ Southern Federal University, \\ 194/2 Stachki Ave., Rostov-on-Don, 344090, Russia \\ email: hizrieva@sfedu.ru
}

\begin{abstract}
For the first time, the complexation of pentacyclic triterpenes of licorice (glycyrrhizic acid (GA) and its aglycone, glycyrrhetinic acid (GLA)) with the novel Russian antiviral drug Camphecene (Camph) was investigated. The complexes obtained at different molar ratios were studied using both UV/Vis spectroscopy and mass spectrometry (ESI MS). Formation of the host:guest complexes were registered: GA and GLA molecular complexes (Camph+2GA; Camph+2GLA) with stability constants $K=6.94 \cdot 10^{6} \mathrm{M}^{-2}$ and $K=2.89 \cdot 10^{6} \mathrm{M}^{-2}$, respectively. The research results demonstrate a considerable potential of ESI MS as a technique for simple and fast detection of formation of the complexes of GA /GLA and the novel drugs.
\end{abstract}

Keywords: Camphecene; glycyrrhizic acid; glycyrrhetinic acid; antiviral activity; supramolecular complexes

Received: 30.10.2020. Accepted: 07.12.2020. Published:30.12.2020.

(C) Khizrieva S. S., Vetrova E. V., Borisenko S. N., Maksimenko E. V., Borisenko N. I., 2020

\section{Introduction}

Influenza is known to be the most common and dangerous respiratory viral infection. It causes annual epidemics and pandemics, leading to significant increases in morbidity and mortality in all regions of the world. In connection with the growing number of cases of viral infections and especially resistant viral strains, it is necessary to improve the available therapeutic methods, complementing them with the discovery of new antiviral agents. On the other fist, it is widely recognized that the medical heritage of plants is a valuable resource for the treatment of infectious disorders. This indicates a growing interest in antiviral products based on sec- ondary plant metabolites [1-3]. The one of the unique plants used in both folk and traditional medicine is licorice. Licorice (Glycyrrhiza glabra L.) is one of the oldest and most widely used medicinal plants [4, 5]. The major plant's metabolite in licorice root is glycyrrhizic acid (GA, 1), pentacyclic triterpene, consisting of one residue of glycyrrhetinic acid (GLA, 2) and two residues of glucuronic acid (Fig. 1).

Licorice triterpenes 1 and 2 are attracting more and more attention of chemists and pharmacologists due to the wide spectrum of their biological activity [6-9]. GA and GLA are active against a wide range of viruses, including herpes, corona, alpha, 


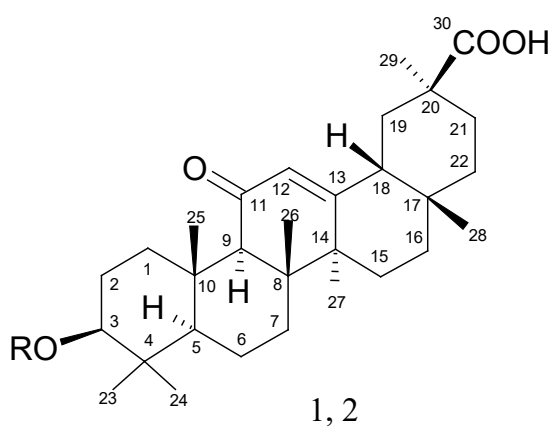

a) GA (1)

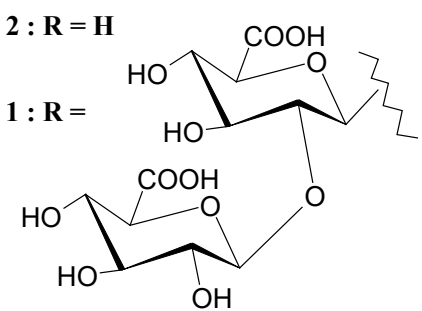

b) GLA (2)

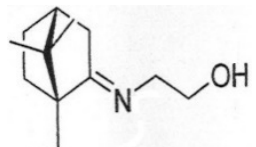

c) Camph (3)

Fig. 1. Formulae of a) glycyrrhetinic acid (2), $\mathrm{C}_{30} \mathrm{H}_{46} \mathrm{O}_{4}$;

b) glycyrrhizic acid (1), $\mathrm{C}_{42} \mathrm{H}_{62} \mathrm{O}_{16}$; c) Camphecene (3), $\mathrm{C}_{12} \mathrm{H}_{21} \mathrm{NO}$

flaviviruses and others [10]. At the same time, GA, GLA and their derivatives exhibit a wide spectrum of antiviral activity both in the form of individual substances $[1,10]$ and in combination with antiviral drugs [11]. GA 1, due to its amphiphilicity, can form supramolecular complexes with various hydrophobic molecules, significantly increasing their solubility. This property was used in the approach of acad. G. A. Tolstikov: to reduce therapeutic doses of drugs and prolong the action [12-13].
In this regard, the presented work considers the possibilities of synthesizing new supramolecular complexes of GA and GLA with new antiviral drug Camphecene for the development of low-dose pharmaceutical substances on their basis. The authors consider that these pharmaceutical substances can be used to suppress the multiplication of viruses in the early stages. Camphecene 3 (Fig. 1), has a broad spectrum of antiviral activity. It is proved to be active against influenza A strains and type B virus [14].

\section{Experimental}

For the research we used GA and $18 \beta$-GLA from Aldrich. Camphecene was synthesized at the Novosibirsk Institute of Organic Chemistry (NIOCH SB RAS) and kindly provided by Prof. N. F. Salakhutdinov. Solvents from Merck, HPLC/MS qualifications. The complexes were obtained by traditional mixing of solutions with different molar ratios of GA, GLA, and Camph in a 70\% aqueous-alcoholic solution. The founding of complexes of GA and GLA with Camph was recorded by UV/Vis spectroscopy using a SPEKS SSP 705 spectrophotometer (190-1100 nm) (manufactured by CJSC

Spectroscopic Systems, Russia). Measurements were performed in a quartz cell. The formation of the complex was considered by the change in optical density at a constant concentration of Camph $(C=$ $0.5 \mathrm{mM}$ ). In order to exclude the contribution of the absorption of triterpenes by GA and GLA, respectively, their absorption spectrum was subtracted from the total spectrum.

The composition of supramolecular complexes of GA and GLA with Camph was investigated with direct injection on a Bruker Daltonics micrOTOF-Q mass spectrometer with electrospray ioniza- 
tion. The micrOTOFcontrol ${ }^{\mathrm{TM}} 2.2$ software was used to analyze the results. The mass spectrometer was calibrated using a Fluka
Electrospray Calibrant Solution. Detection of negative and positive ions was performed in the range of $m / z$ from 50 to 3000 .

\section{Results and discussion}

In accordance with the objectives of the work, sets of complexes of licorice triterpenes GA and GLA with an antiviral drug Camphecene at different molar ratios "guest:host" were synthesized.

In the first step, complexes of GA with Camph were obtained at molar ratios: 1:1 and 2:1 and studied by UV / Vis spectroscopy and mass spectrometry with electrospray ionization. As demonstrated in Fig. 2, with an increase in the concentration of GA from 0 to $1 \mathrm{mM}$, a bathochromic shift of the absorption maximum of Camph $(201 \rightarrow 212 \mathrm{~nm})$ is recorded in the UV / Vis spectra, and a decrease in optical density is observed.

An increase in the concentration of GA (from $1.5 \mathrm{mM}$ and higher) leads to the disappearance of the maximum absorption of Camph, which indicates the complete binding of Camph molecules in the presence of GA (Fig. 3).

From the dependence of the optical density on the concentration of glycyr- rhizic acid (Fig. 3), over the entire range of increase in the concentration of GA, the value of the maximum optical density of Camph decreases.

The study of GLA as a complexing agent demonstrated similarchanges in the UV / Vis spectra of the obtained complexes. When the GLA concentration changes in the range from 0.05 to $0.125 \mathrm{mM}$, a bathochromic shift of the absorption maximum of Camph is recorded (201 $\rightarrow$ $206 \mathrm{~nm})$.

To calculate the stability constant of the complexes, we used the BenesiHildebrand plot (1) [15]. The stability constant of the nGA-Camph complex was estimated from the change in the optical density of Camph $\left(\lambda_{\max }=201 \mathrm{~nm}\right)$ at its fixed concentration in solutions in which the GA (or GLA) concentration was varied. Eq. (1) allows, within the framework of one experiment, not only to estimate the stability constant of the complex $(K)$,

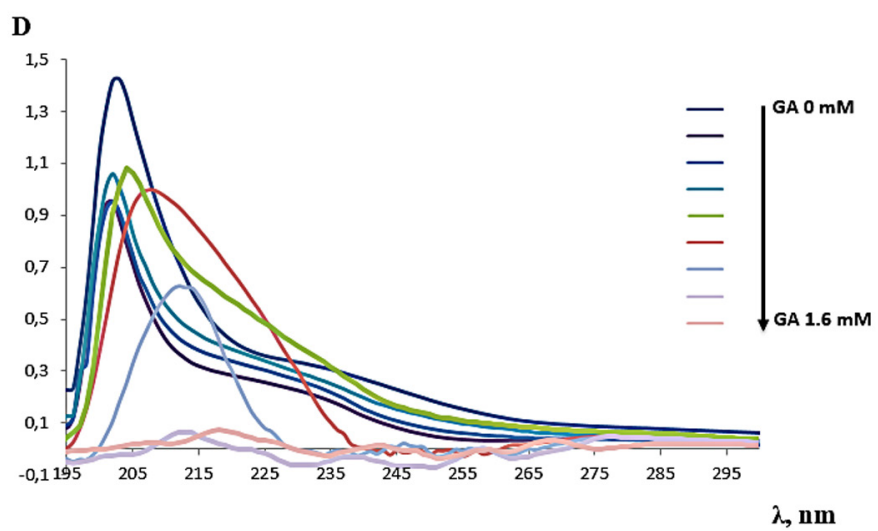

Fig. 2. Absorption spectrum of Camphecene $\left(C_{\text {Camph }}=0.5 \mathrm{mM}\right)$ at different concentrations of GA 
but also to determine the stoichiometry ratio "host:guest" $(n)$ in the complex:

$$
D / \Delta D-1=\left(1 /[\mathrm{GA}]^{n}\right) \cdot(1 / K),
$$

where $\Delta D=\Delta \varepsilon \cdot\left[C_{\text {Camph }}\right]-$ change in the optical density of the solution, and $K$ - the constant of stability of the complex, determined for the reaction:

$$
\begin{aligned}
\text { Camph } & +n \mathrm{GA} \rightleftarrows \text { Camph }-n \mathrm{GA},= \\
& =\frac{[\mathrm{Camph}-n \mathrm{GA}]}{[\mathrm{Camph}] \cdot[\mathrm{GA}]^{n} .}
\end{aligned}
$$

The absorption spectrum of Camph was recorded at a wavelength of $201 \mathrm{~nm}$, while the Camph concentration was constant and amounted to $0.5 \mathrm{mM}$. The obtained dependence of the absorption intensity of Camphecene $(\lambda=201 \mathrm{~nm})$ on the concentration of GA is shown in Fig. 4.

From the slope of the straight-line $D / \Delta D$, depending on $1 /[\mathrm{GA}]^{2}$ (Fig. 4 ), the stability constant of the complex was calculated using Eq. (1). The stability constant for the Camph + GA complex is $1 / K$ $=1.44 \cdot 10^{-7} \mathrm{M}^{2}$ or $K=6.94 \cdot 10^{6} \mathrm{M}^{-2}$.

Recognizing the value of the binding constant, the change in the Gibbs energy was calculated. Obtained from the binding constant, the change in Gibbs energy $\Delta G$ $=-38.4 \mathrm{~kJ}$. Based on the obtained negative value, it can be concluded that the reaction proceeds spontaneously during the formation of GA and Camph complexes. Similarly, according to the Eq. (1), the stability constant of the complex Camph+GLA was calculated, $1 / K=3.46 \cdot 10^{-7} \mathrm{M}^{2}$ or $K=2.89 \cdot 10^{6}$ $\mathrm{M}^{-2}$. Using the value of the binding constant, the change in Gibbs energy was calculated. The change in the Gibbs energy $\Delta G=-36.2$ $\mathrm{kJ}$, which allows us to conclude that the reaction proceeds spontaneously during the formation of a complex of GLA with Camph.

The formation of complexes of Camphecene with natural triterpenoids has also been studied using electrospray ionization mass spectrometry (ESI MS). In the mass spectra of complexes of GA and GLA with Camph in the mode of positive ions, both the peaks of GA, GLA, and Camph ions and the peaks of heterocomplexes containing GA and Camph or GLA and Camph are recorded.

Thus, in the GLA and Camph complexes synthesized both at a ratio of the components of GLA and Camph in 1:1 and at a ratio of 2:1, a peak with $m / z 688.73$ was recorded, corresponding to a single charged ion of the complex GLA and Camph $\left[\mathrm{M}_{\mathrm{GLA}}+\mathrm{M}_{\text {Camph }}+\mathrm{Na}\right]^{+}$. A typical mass spectrum of the GLA-Camph complex is shown in Fig. 5.

The presence of peaks of heterocomplexes containing GA and Camph, GLA and Camph confirm the formation of complexes of GA and GLA with Camph, respectively. The results obtained demon-

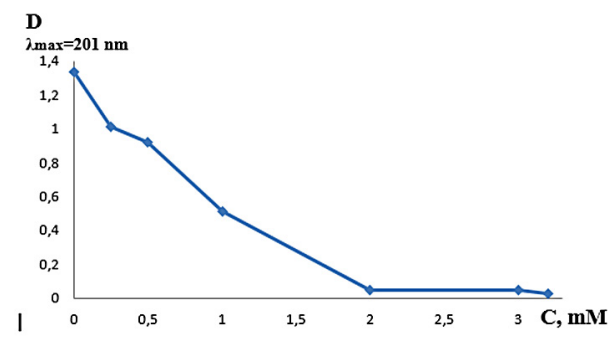

Fig. 3. Dependence of optical density on the concentration of glycyrrhizic acid

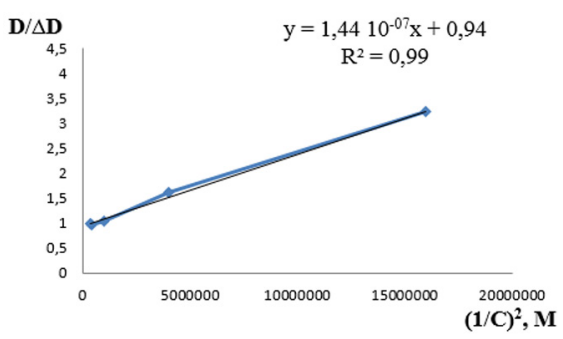

Fig. 4. Dependence of the absorption intensity of Camphecene $(\lambda=201 \mathrm{~nm})$ on the concentration of GA 


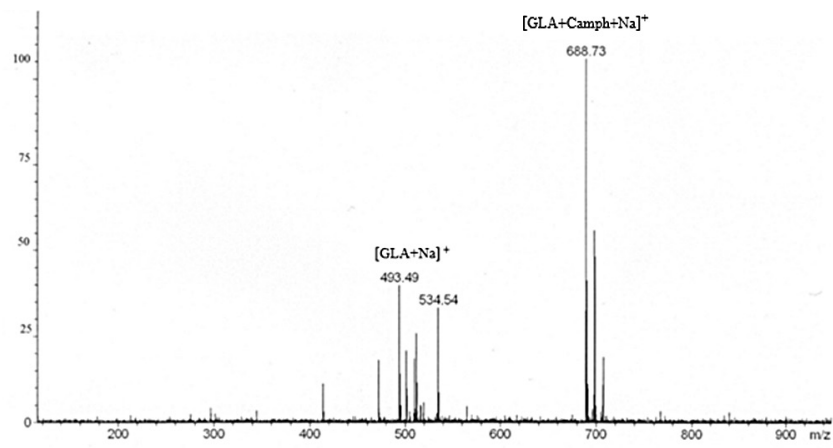

Fig. 5. Mass spectrum of a complex of Camphecene (Camph) + glycyrrhetinic acid (GLA)

strate the high potential of triterpenes GA and GLA in the development of new pharmaceutical forms using the example of Camphecene in the form of molecular complexes.

\section{Conclusions}

In the presented work, complexes of pentacyclic triterpenes of the licorice (glycyrrhizic acid (GA) and glycyrrhetinic acid (GLA), its aglycone) with the antiviral drug Camphecene (Camph) were synthesized and studied.

The complexes obtained at different molar ratios "host - GA (GLA): guest Camph": 1:1 and 2:1 were studied using both UV / Vis spectrophotometry and mass spectrometry with electrospray ionization. Formation of the host:guest complexes were registered: GA and GLA molecules form molecular complexes (Camph+2GA;
Camph+2GLA) with stability constants $K=6.94 \cdot 10^{6} \mathrm{M}^{-2}$ and $K=2.89 \cdot 10^{6} \mathrm{M}^{-2}$, respectively.

In the mode of positive ion, the mass spectra of mixture of GA and GLA with Camph showed peaks of GA, GLA, and Camph ions and the peaks of heterocomplexes containing GA and Camph, and GLA and Camph, respectively. The research results demonstrated the considerable potential of ESI MS as a technique for simple and fast detection of formation of the complexes of GA / GLA and the novel drugs.

\section{Acknowledgements}

This work was supported by the Russian Foundation for Basic Research (RFBR, grant no. 19-33-90211-Aspiranty).

\section{References}

1. Zarubaev VV, Anikin VB, Smirnov VS. Anti-viral activity of glycyrrhetinic and glycyrrhizic acids. Rus J of Inf and Imm = Infektsiyaiimmunitet. 2016;6(3):199-206. doi:10.15789/2220-7619-20163-199-206

2. Akram M, Tahir IM, Shah SMA, Mahmood Z, Altaf A, Ahmad K, Munir N, Daniyal M, Nasir S, Mehboob H. Antiviral potential of medicinal plants against HIV, 
HSV, influenza, hepatitis, and coxsackievirus: A systematic review. Phytother Res. 2018;32(5):811-22.

doi:10.1002/ptr.6024

3. Dhama K, Karthik K, Khandia R, Munjal A, Tiwari R, Rana R, Khurana SK, Sana U, Khan RU, Alagawany M, Farag MR., Dadar M, Joshi SK. Medicinal and Therapeutic Potential of Herbs and Plant Metabolites / Extracts Countering Viral Pathogens Current Knowledge and Future Prospects. Curr Drug Metab. 2018;19(3):236-63. doi:10.2174/1389200219666180129145252

4. Tolstikov GA, Shultz EE, Baltina LA, PokrovskyAG. Glycyrrhizicacid. Rus J of Bioorg Chem [Internet]. 1997;23(9):625-42. Available from: https://europepmc.org/article/ med/9441592

5. Shibata SA. Drug over the millennia: Pharmacognosy, chemistry, and pharmacology of licorice. J YakugakuZasshi. 2000;120(10):849-62.

doi:10.1248/yakushi1947.120.10_849

6. Astafyeva OV, Sukhenko LT, Egorov MA. [Antibacterial activity of extracted biologically active substances and extracts of roots of Glycyrrhiza glabra L]. Khimiya rastitel'nogo syr'ya [Chemistry of raw plant materials]. 2013;(3):261-3. Russian. doi:10.14258/jcprm.1303261

7. Yang R, Wang LQ, Yuan BC, Liu Y. The pharmacological activities of Licorice. Planta Med. 2015;81(18):1654-69.

doi:10.1055/s-0035-1557893

8. Pompei R, Flore O, Marccialis MA, Pani A, Loddo B. Glycyrrhizic acid inhibits virus growth and inactivates virus particles. Nature. 1979;281:689-90. doi:10.1038/281689a0

9. Hussain H, Green IR, Shamraiz U, Saleem M, Badshah A, Abbas G, Rehman NU, Irshad M. Therapeutic potential of glycyrrhetinic acids: a patent review (2010-2017). Expert Opin. Ther. Pat. 2018;28(5):383-98. doi:10.1080/13543776.2018.1455828

10. Baltina LA, Kondratenko RM, Baltina JR, Plyasunova OA, Pokrovskii AG, Tolstikov GA. Search for new drugs: Prospects for the creation of new antiviral drugs based on glycyrrhizic acid and its derivatives. Pharm Chem J. 2009;43(10):539-48. doi:10.1007/s11094-010-0348-2

11. Singh L, Indermun S, Govender M, Kumar P, du Toit LC, Choonara YE, Pillay V. Drug Delivery Strategies for Antivirals against Hepatitis B Virus. Viruses. 2018;10(5):E267. doi:10.3390/v10050267

12. Tolstikova TG, Tolstikov AG, Tolstikov GA. On the way to low-dose medicines. Herald of the Russian Academy of Sciences. 2007;77(10):447-53.

doi:10.1134/S1019331607050012

13. Vetrova EV, Borisenko NI, Lekar’ AV, Maksimenko EV, Borisenko SN, Khizrieva SS. [Mass spectrometry of glycyrrethenic acid complexes with striptomycines]. Khimiya rastitel'nogo syr'ya [Chemistry of raw plant materials]. 2019;(1):119-26. doi:10.14258/jcprm.2019013319

14. Zarubaev VV, Garshinina AV, Tretiak TS, Fedorova VA,Shtro AA, Sokolova AS, YarovayaOI, Salakhutdinov NF. Broad range of inhibiting action of novel Camphor- 
based compound with anti-hemagglutinin activity against influenza viruses in vitro and in vivo. Antiviral Res. 2015;120:126-33.

doi:10.1016/j.antiviral.2015.06.004

15. Vavilin VA, Salakhutdinov NF,RaginoYuI, Polyakov NE, Taraban MB, Leshina TV, Stakhneva EM, Lyakhovich VV, NikitinYuP, Tolstikov GA. The Cholesterol Lowering Properties of the Complex Compound Simvastatin with Glycyrrhizic Acid (Simvaglyzin) in Experimental Models. Biochem (Moscow) Suppl Series B: Biomed Chem. 2008;2(4):373-80.

doi:10.1134/S1990750808040070 